\title{
Physical Activity and Function in Older, Long-term Colorectal Cancer Survivors
}

\author{
Brent L. Johnson ${ }^{1,2}$, Amy Trentham-Dietz ${ }^{2,3}$, Kelli F. Koltyn ${ }^{1}$, and Lisa H. Colbert ${ }^{1,3}$ \\ ${ }^{1}$ Department of Kinesiology, University of Wisconsin, Madison, WI \\ ${ }^{2}$ Department of Population Health Sciences, University of Wisconsin, Madison, WI \\ ${ }^{3}$ Paul P. Carbone Comprehensive Cancer Center, University of Wisconsin, Madison, WI
}

\section{Abstract}

Objective-Increasing age and cancer history are related to impaired physical function. Since physical activity has been shown to ameliorate age-related functional declines, we evaluated the association between physical activity and function in older, long-term colorectal cancer survivors.

Methods-In 2006-2007, mailed surveys were sent to colorectal cancer survivors, aged $\geq 65$ years when diagnosed during $1995-2000$, and identified through a state cancer registry. Information on physical activity, physical function and relevant covariates was obtained and matched to registry data. Analysis of covariance and linear regression were used to compare means and trends in physical function across levels of activity in the final analytic sample of 843 cases.

Results-A direct, dose-dependent association between physical activity and function was observed ( $\mathrm{p}_{\text {trend }}<.001$ ), with higher SF-36 physical function subscores in those reporting high vs. low activity levels ( $65.0 \pm 1.7$ vs. $42.7 \pm 1.7$ (mean \pm standard error)). Walking, gardening, housework, and exercise activities were all independently related to better physical function. Moderate-vigorous intensity activity $\left(\mathrm{p}_{\text {trend }}<.001\right)$ was associated with function, but light activity ( $\mathrm{p}_{\text {trend }}=0.39$ ) was not.

Conclusion-Results from this cross-sectional study indicate significant associations between physical activity and physical function in older, long-term colorectal cancer survivors.

\section{Keywords}

exercise; walking; physical function; elderly

\section{Introduction}

The number of cancer survivors living in the United States has grown substantially from the 3.0 million estimated in 1971 to more than 10.5 million today [1;2]. As the demography of the U.S. population shifts toward increasing age, the population of survivors will continue to grow. By current rates, one out of every two men and one out of every three women in this country will be diagnosed with some form of cancer in their lifetime [3]. Given improved detection and treatment, nearly $66 \%$ of patients diagnosed today will live five years past diagnosis, many of whom will live 10-20 or more years beyond their cancer diagnosis [1;2].

Corresponding Author: Lisa H. Colbert, PhD, MPH, Dept. of Kinesiology, 2000 Observatory Dr., Madison, WI 53706, Ph: 608-265-5946, Fax: 608-262-1656, E-mail: E-mail: 1hcolbert@education.wisc.edu. 
While substantial research has been conducted in cancer detection and prevention, less information is available regarding cancer patients post-treatment. Studies suggest that cancer survivors are at increased risk for functional limitation compared to similarly aged individuals with no cancer history [4-17]. Hewitt, et al.[4] found that cancer survivors were at a nearly two-fold increased risk for functional limitation; however, in the presence of another chronic illness, this jumped to a 5-fold increase in risk. Chirikos et al. [11] found that the consequences of functional limitation associated with breast cancer diagnosis extended beyond quality of life, exacting substantial financial consequences on the patients, their families and the economy at large.

Physical activity is one intervention modality that has been effective in combating functional decline in older adults. Evidence suggests that older adults accumulating 150 minutes of moderate intensity activity per week, as recommended by the U.S. federal government[18], can reduce their risk of functional limitation by up to 50\% [19-21]. Consequently, several recent population-based studies [22;23] and physical activity intervention trials [24-30] have been conducted among breast [22-26;28-30], prostate [23;24], and colorectal [27] cancer survivors in an attempt to ameliorate functional declines and other quality of life changes associated with cancer diagnosis and treatment. While these studies have obtained mixed results, they have primarily focused on short-term survivors [23-28], middle aged adults [22; $25 ; 26 ; 28-30]$, and breast cancer, specifically [22-26;28-30]. Given the differences by cancer site in patient characteristics and treatment regimens, it is possible that the relationships between activity and function my differ, and thus it is important to explore some of the more common cancer sites that have received little attention [31;32].

Specifically, little is known about the association between physical activity and function in older, long-term colorectal cancer survivors. As roughly $60 \%$ of all cancer survivors are aged $\geq 65$ years [2], and age is an independent risk factor for functional decline [33-36], it is increasingly important that we understand the association between physical activity and function within this high risk population. The purpose of this study was to examine the association between physical activity and function in a sample of older, long-term colorectal cancer survivors from the state of Wisconsin. We also explored effect modification of this association by sex and age.

\section{Materials and Methods}

\section{Subjects}

Subjects were drawn from the Wisconsin Cancer Reporting System (WCRS), a statewide mandatory tumor registry. From 1995-2000, 5868 cases of colorectal cancer were diagnosed in adults aged 65-84 yrs and reported to the registry, of which all cases from Dane county and a random $\sim 40 \%$ of non-Dane county cases were sampled. Of the 2354 cases sampled, 586 were deceased according to the Social Security Death Index, resulting in 1768 potential participants.

\section{Study Design}

For this cross-sectional study, the 1768 potential participants were sent an introductory letter and study survey by mail directly from the investigators along with a five dollar incentive to complete the survey. Non-respondents were followed up with reminder post cards, a second letter and survey, and finally, telephone calls. During the call they could either decline to participate, request another mailing of the survey, or choose to complete the survey over the phone. Additional information was collected from the statewide tumor registry including sex, birth date, diagnosis date, stage, grade, and treatment information. The study protocol was approved by the University of Wisconsin Institutional Review Board. 
Of the original 1768 potential participants who were mailed surveys, 938 (53\%) completed the survey either by mail $(97 \%)$ or telephone (3\%). Three hundred ninety nine $(23 \%)$ refused participation, $305(17 \%)$ could not be located by mail or contacted by phone, $61(3 \%)$ were too ill to complete the questionnaire, 41 (2\%) were reported as deceased, and $24(1 \%)$ were unable to participate for other reasons. Therefore, among persons who we were able to contact, the response rate was $64 \%$. Data from the statewide cancer registry indicated that nonrespondents were more likely to be female, older, diagnosed at a later age, and further removed from diagnosis when compared to respondents.

\section{Survey}

The survey incorporated standard measures of physical activity and physical function in addition to demographic, lifestyle, and health history questionnaires. The Community Healthy Activities Model Program for Seniors (CHAMPS) physical activity questionnaire [37] is a 41item questionnaire assessing frequency and duration of a broad range of activities typically engaged in by older adults including leisure-time activities, walking, household activities, and exercise. The survey questions focus on a typical week during the past four weeks. Respondents reply with the number of times and total hours per week spent in that activity. MET-hour/week (MET-hr/wk) equivalents are calculated as recommended, and body weight was used to calculate activity in kcal/wk [37]. Summary scores are presented for total physical activity, moderate-vigorous activity (activities with MET values $\geq 3.0$ ), and for light activities (MET values $<3.0)$. This survey has been found to be reliable $(\mathrm{ICC}=0.66)$ and valid for use in older adults [38].

The Medical Outcomes Study 36-Item Short-Form Health Status Survey (SF-36) is a widely used generic measure of health. It has been shown to be reliable and valid in multiple populations, including cancer survivors [39-43]. The SF-36 consists of eight subscales of health including physical functioning (possible scoring range 0-100), which focuses on the degree of limitation in specific activities including: vigorous activities; moderate activities; lifting or carrying groceries; climbing several flights of stairs; climbing one flight of stairs; bending, kneeling or stooping; walking more than a mile; walking several blocks; walking one block; and bathing or dressing yourself. Descriptive information collected from the survey included education attained, tobacco use (current and ever) and current alcohol consumption. The brief health history inquired about 17 conditions and diseases that the participant might currently have, and subjects also reported their height and weight. Information on all previous cancer diagnoses (site, year of diagnosis, and treatment for most recent cancer) was collected as well as any falls or hospitalizations in the previous 12 months. Participants were also asked if they currently had any pain beyond minor everyday types of pain such as headaches. The tumor registry was used to obtain information on age, tumor site, stage, grade, and treatment.

\section{Analyses}

Missing responses to individual CHAMPS questions were coded as no activity; however, if participants answered none of the physical activity questions, their score was set to missing. Co-morbidities were summed from positive responses to the current health conditions inventory. Cancer treatment information from both the registry and self-report were combined, and binary variables of any indication of a specific treatment vs. no indication were created for surgery, chemotherapy, and radiation. In order to examine the physical function association by intensity of activity, we examined associations with light and moderate-vigorous activity separately. We also examined type of activity by categorizing individual questions into the following categories: housework, gardening, any walking, exercise/recreation/sports, and stretching. In separate analyses, we separated activities into categories of aerobic (skating, jogging, walking uphill, brisk walking, biking, aerobic machines, swimming fast, and aerobic dance), resistance (heavy housework, heavy gardening, non-swimming water exercises, heavy 
strength training and light strength training), and an "other" activity category (eg. dancing, golf, tennis). The physical function subscale was used in the current analyses, and the standard SF-36 scoring method was used [42-44]. Individuals were excluded from the study analyses if the entire physical activity or comborbidity sections were missing, or if their physical function subscale score was missing.

Means and standard deviations of covariates across quartiles of physical activity were calculated using analysis of variance for continuous variables. Categorical variables were evaluated using Mantel-Haenszel chi-square or Fisher's exact test for analyses with cell sizes $\leq 5$. Analysis of covariance (ANCOVA) was used to examine the association of physical activity and physical function. Physical activity was categorized by quartile for the main analysis and analysis by intensity, and by categories of none, low, moderate, and high activity based on the cohort-distribution for specific activity categories. Medians and interquartile ranges are presented for physical activity, where appropriate, due to non-normal distributions. Covariates considered for these analyses included age, sex, education, current smoking status, alcohol consumption, body mass index (BMI), body weight, type and number of comorbidities, pain, hospitalizations and falls in the previous 12 months, number of cancer diagnoses, stage of diagnosed cancer, age at diagnosis, time since diagnosis, and cancer treatment type. Age and sex were included as covariates in all analyses (model 1). Other covariates that changed the significance of the physical activity term or appreciably reduced the magnitude of difference between the least and most active quartiles were included in a second model (model 2), including: age, sex, education (less than high school (HS), HS, more than HS), alcohol consumption (none, occasional, 1-7 drinks/wk, >7 drinks/wk), BMI (<20, 20-24.9, 25-29.9, $\geq 30 \mathrm{~kg} / \mathrm{m}^{2}$ ), pain (yes, no), hospitalizations, (yes, no), falls (yes, no), number of comorbidities $(0,1,2,3,4, \geq 5)$. ANCOVA was used to calculate least squares means across categories of activity, and tests for linear trend were performed using an ordered categorical variable based on the median activity value in each category. Effect modification by age and sex were tested using cross-product terms of the bivariate age or sex variable and the scored quartile variable for physical activity, and in separate models, stratified analysis. All analyses were conducted using SAS statistical software (SAS Institute, Inc., Cary, NC).

\section{Results}

From the 938 surveys returned, 95 responses were excluded for the following reasons: 11 were currently undergoing cancer treatment, 28 were missing physical activity data, 8 were missing physical function data, and 48 were missing information on relevant covariates. Complete data was obtained for 843 individuals for whom the analyses were conducted. The mean age of respondents was 81.5 years (SD, 5.8), and on average, they were $8.2 \pm 1.7 \mathrm{yrs}$ past their diagnosis. Ninety seven percent of participants reported receiving surgery for their cancer, $35 \%$ reported receiving chemotherapy, and 18\% reported receiving radiation. Given the high prevalence of surgery, only chemotherapy and radiation were considered as potential covariates, and neither were related to the physical function subscore nor did they confound the models. The sample was $48 \%$ male and $99 \%$ white. These individuals reported a median energy expenditure of 2135 kilocalories/ week (IQR: 935 - 3821) and mean physical function score of 55.0 (SD, 30.5). Sample characteristics by level of physical activity are displayed in Table 1. Subjects in the lowest quartiles of total physical activity did predominantly light intensity activity. As the total activity level increased, so did the time spent on moderatevigorous activity. Compared to the least active quartile, the most active individuals tended to be younger, more highly educated, and male. These more active individuals also reported drinking more alcohol, having fewer comorbidities, were less likely to have fallen in the past 12 months and/or to have been hospitalized in the past 12 months, and were less likely to report pain beyond normal levels than the least active respondents. 
A direct, dose-dependent association between total physical activity and function was observed within this population $\left(\mathrm{p}_{\text {trend }}<.001\right)$ (Table 2 ). The mean level of physical function among the most active was more than 25 points higher than that of the least active $(\mathrm{p}<.001)$ after controlling for age, sex, education, alcohol consumption, and BMI. Further adjustment for pain, hospitalizations, falls and comorbidities attenuated the association only slightly, with the difference in function between low and high categories of physical activity still 22 points. Analyses by intensity of activity indicate that the relationship with total physical activity is largely driven by moderate-vigorous activity rather than light activity, as the relationship between moderate-vigorous activity and function is similar to that of total activity and unaffected by adjustment for light activity $(\mathrm{p}<0.001)$. In contrast, there is no signficicant relationship with light activity after adjustment for moderate-vigorous activity $(\mathrm{p}=0.39)$. On average, women reported lower levels of physical functioning than men across all levels of physical activity; however, no significant interaction by sex was observed $(\mathrm{p}=0.39)$, with the same direct association seen in both men ( $\left.\mathrm{p}_{\text {trend }}<.001\right)$ and women $\left(\mathrm{p}_{\text {trend }}<.001\right)$ (Table 3$)$. Similarly, although function was much lower among those who were older in the population ( $>$ median of $80.7 \mathrm{yrs}$ at the time of survey), there was no significant interaction by age ( $\mathrm{p}=0.60)$, with the same dose-response relationship with physical activity seen among both the younger ( $\left.\mathrm{p}_{\text {trend }}<0.001\right)$ and older $\left(\mathrm{p}_{\text {trend }}<0.001\right)$ study participants (Table 3$)$.

When examined by type of exercise, only stretching was not associated with physical function in the anticipated direction (Table 4). Participation in housework, gardening, and walking were all strongly associated with higher levels of physical function ( $\mathrm{p}_{\text {trend }}<0.001$ ). SF-36 function scores were lowest in those who didn't participate in those activities, and for housework and gardening, there was little to no added benefit of doing more; however for walking, function scores increased across levels of activity. While participation in exercise/recreation/sport was associated with better function ( $\left.\mathrm{p}_{\text {trend }}=0.004\right)$, only the highest level of activity had a significantly better function score compared to those who did no activity of this type. Stretching was not associated with physical function $\left(\mathrm{p}_{\text {trend }}=0.69\right)$. When we used the classification of aerobic and resistance activity, both were similarly associated with physical function ( $\mathrm{p}_{\text {trend }}$ $<0.001$ ). Individuals reporting the greatest amount of participation in aerobic activities scored 10 points higher on the physical function subscale than individuals reporting no participation in aerobic activities (SF-36 score of 61.4 vs. 51.4, p<.001). Similarly, those reporting the greatest amount of resistance-type activities scored 10 points higher than those doing no resistance activities (SF-36 score of 59.4 vs. 48.6, p<0.001). These relationships persisted with adjustment for the other types of activity.

\section{Discussion}

Physical activity was directly associated with physical functioning $(\mathrm{p}<.001)$ in this sample.

When accounting for potential mediating variables such as recent falls and comorbidities, the associations were only modestly attenuated. Neither sex nor age modified the association with similarly strong associations seen in men and women, and in those both under and over the age of 80 years. Moderate-vigorous intensity activity appeared to be the important component of total activity, as light activity alone was not associated with physical function. Activity of many types, including housework, gardening, walking, and exercise appeared to be related to better function; however, stretching was not.

Participants reported engaging in $2773 \pm 2586 \mathrm{kcals} / \mathrm{wk}$ of physical activity, on average, however, the median level of physical activity reported was $2135 \mathrm{kcal} / \mathrm{wk}$. Stewart, et al. [37] previously reported a mean energy expenditure of $2420(\mathrm{SD}, 1831)$ as measured by the CHAMPS questionnaire in a sample of men and women aged 65-90 yrs, which appears reasonably consistent with our findings. This volume of activity appears quite high given that most adults do not meet the $1000 \mathrm{kcal} / \mathrm{wk}$ recommendation by the CDC/ACSM [45;46]. To 
some extent, this potential overreporting could be explained by the inclusion of light intensity activities on the CHAMPS questionnaire; however, even the moderate-vigorous activity reported appears quite high. Regardless, if the potential over-reporting is consistent across activity levels it should not affect our ability to compare low vs. high activity groups. Another important possibility though, is that we have selection bias in our sample and that we were more likely to receive responses from survivors who do more physical activity. Conclusions regarding the general population of older colorectal cancer survivors must thus be tempered with this potential selection bias in mind.

The mean Physical Functioning (PF) subscale score of our participants $(55.0 \pm 30.5)$ is close to the SF-36 age-based population norms of men and women aged $\geq 75$ years $(53.20 \pm 29.98)$. A previous study of long-term female colorectal cancer survivors in Wisconsin reported a mean PF score of $62.4 \pm 25.8$ for a group of 81 women aged 65-85 years [47], which again appears consistent with the results of this study when considering the differences in age and sex. We observed a very strong and significant 22 -point difference in function between people reporting the lowest vs. the highest levels of total physical activity, and a 21-point difference across levels of moderate-vigorous activity. This difference is more marked than the 15.4 point difference between regular vigorous exercisers and non-regular vigorous exercisers previously reported by Demark-Wahnefried, et al.[23] in a group of breast and prostate cancer survivors at $\sim 18$ months post-diagnosis. This inconsistency may be due to the difference in physical activity assessment, with inclusion of moderate activity in our moderate-vigorous score vs. the focus on vigorous activity in the study by Demark-Wahnefried et al.[23]. Consequently, their non-regular vigorous exercisers may have included people doing more moderate activity, thus reducing the potential difference in function. Importantly, our study suggests the largest difference in physical function between two consecutive activity groups is seen between the 1 st and 2 nd quartiles. This suggests that movement from the least active group to just a modest activity level is associated with the largest change in physical function. Additionally, we saw very strong relationships with housework, gardening, and walking, suggesting that exercise, per se, may not be necessary to maintain physical function at higher levels.

Ware [42] has argued that a 5-10 point difference on any of the eight SF-36 subscales is clinically relevant, thus, the 10 and 12-point differences observed between the least active group and the published age norm and the overall sample mean, respectively, suggest a clinically meaningful functional impairment in these individuals. Conversely, the most active men and women report PF levels nearly 12 and 10 points higher than the published age norm and sample mean, respectively. In essence, these most active individuals report functional status similar to that of individuals aged 10 years younger [42], further reinforcing the notion that physical activity and functional ability are highly related in older adults.

To our knowledge, this is the first study to examine sex differences in the association of physical activity and function among cancer survivors. We hypothesized that women would show a stronger association between physical activity and function, considering that women tend to report both more numerous and more severe limitations than do men [4]. Though women did report lower mean functional status at all levels of activity, the association of physical activity and function did not vary by sex. We also hypothesized that the physical activity and function association may be modified by age, and that some of our oldest subjects would not have the same, direct relationship. We divided our subjects into two groups based on the median age of our sample of 80.7 years, and found the same strong relationship among those aged 65-80.7 as among those aged 80.7-103 years of age. Our data suggest that physical activity may be beneficial at any age.

One benefit of observational studies over intervention studies is the ability to examine the natural distribution in types and amounts of activity that are done in given populations. In 
particular, it is useful to know if non-exercise activities such as housework or gardening are associated with better physical function. We used the CHAMPS physical activity questionnaire to categorize responses to specific questions into general categories of activities that people frequently report participating in such as housework, gardening, and walking. When mutually adjusted, all three of these categories of activity, in addition to more traditionally examined exercise activities, were significantly associated with better function. Importantly, for housework, gardening, and exercise, people doing any activity of those types had significantly better function when compared to those who did none. With walking, function improved with increasing amounts of activity. Perhaps not too surpisingly, stretching was unrelated to physical function.

Lifestyle-based [24;30], resistance-based [28], aerobic-based [25-27], and combined aerobic and resistance-based [29] exercise interventions have been conducted in breast, prostate and colorectal cancer survivors. While the majority of these interventions have shown significant improvements in functional measures [25;26;28-30], no study has compared the effects of resistance vs. aerobic activities on physical function in cancer survivors. We hypothesized that resistance activities would be more strongly associated with function than aerobic activities, assuming that loss of strength following treatment, particularly related to surgery and/or chemotherapy, would be more functionally limiting than loss of aerobic capacity. However, no differences in function were seen based on the type of activity after controlling for other energy expenditure and relevant covariates. This may be a result of examining a composite physical function score rather than looking at task-specific function (e.g. carrying a load vs. walking $1 / 4$ mile). Cancer treatment did not appear related to physical function in our analyses suggesting that there may not be long-term consequences of either chemotherapy or radiation treatment in colorectal cancer patients who have lived this long. Given the potential errors in our treatment measures, which were not directly taken from medical records, further investigation into this issue is warranted.

Strengths of this study include a large sample size, including both men and women who had the same primary cancer. We used validated measures of both physical activity and function. Additionally, the use of the CHAMPS physical activity questionnaire allowed us to examine the potential difference between intensity and types of activity. Limitations of this study include the cross-sectional design, self-reported measures of activity and function, and limited generalizability. Given the study design, we cannot attribute higher levels of function to physical activity participation; however, activity and function remained strongly associated even after including several potential markers of lower functional status (eg. comorbidities, falls, hospitalizations, pain) in the model, suggesting some independent relation of activity and function. As with all self-report surveys, our measures may be subject to recall bias, and as previously mentioned, we may also have some selection bias. Finally, the modest response rate (65\%) accompanied by the lack of racial and ethnic diversity in this sample limits the generalizability of our findings.

Though residual confounding cannot be ruled out, and the results of this study do not overtly suggest that physical activity improves function in older cancer survivors, taken in context with data from physical activity intervention trials [24-30] and other population-based studies [22;23], the overall evidence suggests that physical activity may be one avenue for improving physical function in long-term cancer survivors. Our results suggest that the potential benefits would be apparent for both men and women, for people choosing to participate in a variety of activities, and more so for participation in moderate-vigorous activities than light intensity activities. Further studies are needed to identify characteristics of a physical activity intervention program that would be safe, promote high levels of adherence, and maximize functional benefits among older cancer survivors. 


\section{Acknowledgements}

Laura Stephenson and the staff of the Wisconsin Cancer Reporting System; Jane McElroy, Julie McGregor, John Hampton, and Maggie Perdzock of the UW Comprehensive Cancer Center Survey Research Shared Service; Masataka Umeda, LeeAnne Flygt and Ann Wiringa for assistance with data collection and entry.

Acknowledgment of financial support: Support for this research was provided by the Aging and Cancer Program, University of Wisconsin Paul P. Carbone Comprehensive Cancer Center through NIH grant numbers P20 CA103697 and P30 CA14520.

\section{References}

1. Centers for Disease Control. Cancer Survivorship ---United States, 1971-2001. MMWR 2004;53:526529. [PubMed: 15215740]

2. National Cancer Institute Office of Cancer Survivorship. 2004. http://dccps.nci.nih.gov/ocs/ocs_factsheet.pdf

3. American Cancer Society. Cancer Facts and Figures 2006. Atlanta: American Cancer Society; 2006.

4. Hewitt M, Rowland J, Yancik R. Cancer survivors in the United States: age, health, and disability. J Gerontol Med Sci 2003;58:82-91.

5. Demark-Wahnefried W, Aziz N, Rowland J, Pinto B. Riding the crest of the teachable moment: promoting long-term health after the diagnosis of cancer. J Clin Oncol 2005;23:5814-5830. [PubMed: 16043830]

6. Sweeney C, Schmitz K, Lazovich D, Virnig B, Wallace R, Folsom A. Functional limitations in elderly female cancer survivors. J Natl Cancer Inst 2006;98:521-529. [PubMed: 16622121]

7. Schroevers M, Ranchor A, Sanderman R. Adjustment to cancer in the 8 years following diagnosis: a longitudinal study comparing cancer survivors with healthy individuals. Social Sci Med 2006;63:598610 .

8. Ramsey S, Andersen M, Etzioni R, Moinpour C, Peacock S, Potosky A, Urban N. Quality of life in survivors of colorectal carcinoma. Cancer 2000;88:1294-1303. [PubMed: 10717609]

9. Given B, Given C, Azzouz F, Stommel M. Physical functioning of elderly cancer patients prior to diagnosis and following initial treatment. Nurs Res 2001;50:222-232. [PubMed: 11480531]

10. GIven C, Given B, Azzouz F, Stommel M, Kozachik S. Comparison of changes in physical functioning of elderly patients with new diagnoses of cancer. Med Care 2000;38:482-493. [PubMed: 10800975]

11. Chirikos T, Russell-Jacobs A, Jacobsen P. Functional impairment and the economic consequences of female breast cancer. Women Health 2002;36:1. [PubMed: 12215000]

12. Keating N, Nørredam M, Landrum M, Huskamp H, Meara E. Physical and mental health status of older long-term cancer survivors. J Am Geriatr Soc 2005;53:2145-2152. [PubMed: 16398900]

13. Kurtz M, Kurtz J, Stommel M, GIven C, Given B. Loss of physical functioning among geriatric cancer patients: relationships to cancer site, treatment, and comorbidity and age. Eur J Cancer 1997;33:23522358. [PubMed: 9616281]

14. Ganz P, Kwan L, Stanton A, Krupnick J, Rowland J, Meyerowitz B, Bower J, Belin T. Quality of life at the end of primary treatment of breast cancer: first results from the moving beyond cancer randomized trial. J Natl Cancer Inst 2004;96:376-387. [PubMed: 14996859]

15. Ganz P, Desmond K, Leedham B, Rowland J, Meyerowitz B, Belin T. Quality of life in long-term, disease-free survivors of breast cancer: a follow-up study. J Natl Cancer Inst 2002;94:39-49. [PubMed: 11773281]

16. Mols F, van de Poll-Franse L, Vinberhoets A, Hendrikx A, Aaronson N, Houterman S, Coebergh J, Essink-Bot M. Long-term quality of life among Dutch prostate cancer survivors: results of a population-based study. Cancer 2006;107:2186-2196. [PubMed: 17013914]

17. Anthony T, Jones C, Antoine J, Sivess-Franks S, Turnage R. The effect of treatment for colorectal cancer on long-term health-related quality of life. Ann Surg Oncol 2001;8:44-49. [PubMed: 11206224]

18. U.S.Department of Health and Human Services. 2008 Physical Activity Guidelines for Americans. US Department of Health and Human Services; 2008. 
19. Visser M, Simonsick E, Colbert L, Brach J, Rubin S, Kritchevsky S, Newman A, Harris T. for the Health ABC Study. Type and intensity of activity and risk of mobility limitation: the mediating role of muscle paramters. J Am Geriatr Soc 2005;53:762-770. [PubMed: 15877550]

20. Cress M, Buchner D, Questad K, Esselman P, deLateur B, Schwartz R. Exercise: effects on physical functional performance in independent older adults. J Gerontol A Biol Med Sci 1999;54:M242M248.

21. Brach J, Simonsick E, Kritchevsky S, Yaffe K, Newman A. for the Health AaBCSRG. The association between physical function and lifestyle activity and exercise in the Health, Aging and Body Composition Study. J Am Geriatr Soc 2004;52:502-509. [PubMed: 15066063]

22. Kendall A, Mahue-Giangreco M, Carpenter C, Ganz P, Bernstein L. Influence of exercise activity on quality of life in long-term breast cancer survivors. Qual Life Res 2005;14:361-371. [PubMed: 15892425]

23. Demark-Wahnefried W, Clipp E, Morey M, Pieper C, Sloane R, Snyder D, Cohen H. Physical function and associations with diet and exercise: Results of a cross-sectional survey among elders with breast or prostate cancer. Int J Beh Nutr and Phy Act 2004:1-16.

24. Demark-Wahnefried W, Clipp E, Morey M, Pieper C, Sloane R, Clutter Snyder D, Cohen H. Lifestyle intervention development study to improve physical function in older adults with cancer: outcomes from Project LEAD. J Clin Oncol 2006;24:3465-3473. [PubMed: 16849763]

25. Courneya K, Mackey J, Bell G, Jones L, Field C, Fairey A. Randomized controlled trial of exercise training in postmenopausal breast cancer survivors: cardiopulmonary and quality of life outcomes. J Clin Oncol 2003;21:1660-1668. [PubMed: 12721239]

26. Segal R, Evans W, Johnson D, Smith J, Colletta S, Gayton J, Woodard S, Wells G, Reid R. Structured exercise improves physical functioning in women with Stages I and II breast cancer: results of a randomized controlled trial. J Clin Oncol 2001;19:657-665. [PubMed: 11157015]

27. Courneya K, Friedenreich C, Quinney H, Fields A, Jones L, Fairey A. A randomized trial of exercise and quality of life in colorectal cancer survivors. Eur J Cancer Care 2003;12:347-357.

28. McKenzie D, Kalda A. Effect of upper extremity exercise on secondary lymphedema in breast cancer patients: a pilot study. J Clin Oncol 2003;21:463-466. [PubMed: 12560436]

29. Herrero F, San Juan A, Fleck S, Balmer J, Pérez M, Cañete S, Earnest C, Foster C, Lucía A. Combined aerobic and resistance training in breast cancer survivors: a randomized, controlled pilot trial. Int $\mathrm{J}$ Sports Med 2006;27:573-580. [PubMed: 16802254]

30. Basen-Engquist K, Carmack Taylor C, Rosenblum C, Smith M, Shinn E, Greisinger A, Gregg X, Massey P, Valero V, Rivera E. Randomized pilot test of a lifestyle physical activity intervention for breast cancer survivors. Patient Educ Counsel 2006;64:225-234.

31. Courneya K, Friedenreich C. Physical exercise and quality of life following cancer diagnosis: a literature review. Ann Behav Med 1999;21:171-179. [PubMed: 10499138]

32. Stevinson C, Lawlor D, Fox K. Exercise interventions for cancer patients: systematic review of controlled trials. Cancer Causes Control 2004;15:1035-1056. [PubMed: 15801488]

33. Singh M. Exercise and aging. Clin Geriatr Med 2004;20:201-221. [PubMed: 15182878]

34. Roubenoff R, Hughes V. Sarcopenia: current concepts. J Gerontol A Biol Med Sci 2000;55:M716M724.

35. Doherty T. The influence of aging and sex on skeletal muscle mass and strength. Curr Opinion Clin Nutr Metab Care 2001;4:503-508.

36. Hawkins S, Wiswell R. Rate and mechanism of maximal oxygen consumption decline with aging: implications for exercise training. Sports Med 2003;33:877-888. [PubMed: 12974656]

37. Stewart A, Mills K, King A, Haskell W, Gillis D, Ritter P. CHAMPS physical activity questionnaire for older adults: outcomes for interventions. Med Sci Sports Exerc 2001;33:1126-1141. [PubMed: 11445760]

38. Harada N, Chiu V, King A, Stewart A. An evaluation of three self-report physical activity instruments for older adults. Med Sci Sports Exerc 2001;33:962-970. [PubMed: 11404662]

39. Reulen R, Zeegers M, Jenkinson C, Lancashire E, Winter E, Jenney M, Hawkins M. The use of the SF-36 questionnaire in adult survivors of childhood cancer: evaluation of data quality, score reliability, and scaling assumptions. Health Qual Life Outcomes 2006;4:77. [PubMed: 17022814] 
40. Mosconi P, Cifani S, Crispino S, Fossati R, Apolone G. Head and Neck Cancer Italian working group. The performance of SF-36 health survey in patients with laryngeal cancer. Head Neck 2000;22:175182. [PubMed: 10679906]

41. Pinar R. Reliability and construct validity of the SF-36 in Turkish cancer patients. Qual Life Res 2005;14:259-264. [PubMed: 15789959]

42. Ware, JJ.; Snow, K.; Kosinski, M.; Gandek, B. SF-36 Health Survey Manual and Interpretation Guide. Boston, MA: The Health Institute, New England Medical Center; 1993.

43. Ware, JJ.; Kosinski, M.; Keller, S. SF-36 Physical and Mental Health Summary Scales: A User's Manual. Boston, MA: Health Assessment Lab, New England Medical Center; 1994.

44. Ware JJ, Sherbourne C. The MOS 36-Item Short Form Health Survey (SF-36): I. Conceptual framework and item selection. Med Care 1992;30:473-483. [PubMed: 1593914]

45. Pate R, Pratt M, Blair S, Haskell W, Macera C, Bouchard C, Buchner D, Ettinger W, Heath G, King A, Kriska A, Leon A, Marcus B, Morris J, Paffenbarger R Jr, Patrick K, Pollock M, Rippe J, Sallis J, Wilmore J. Physical activity and public health. A recommendation from the Centers for Disease Control and Prevention and the American College of Sports Medicine. JAMA 1995;273:402-407. [PubMed: 7823386]

46. Arriaza Jones D, Ainsworth B, Croft J, Macera C, Lloyd E, Yusuf H. Moderate leisure-time physical activity. Who is meeting the public health recommendations? A national cross-sectional study. Arch Fam Med 1998;7:285-289. [PubMed: 9596466]

47. Trentham-Dietz A, Remington P, Moinpour C, Hampton J, Sapp A, Newcomb P. Health-related quality of life in female long-term colorectal cancer survivors. Oncologist 2003;8:342-349.

[PubMed: 12897331] 
Johnson et al.

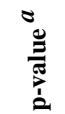

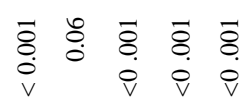

$\stackrel{0}{\circ}$

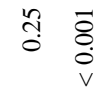

$\overline{0}$.
$\dot{0}$
$\dot{v}$
$\dot{v}$

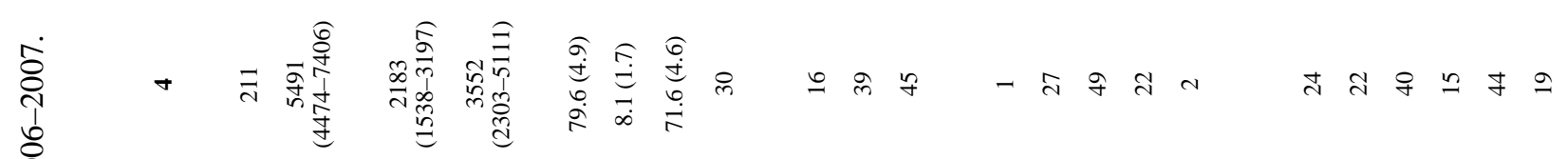

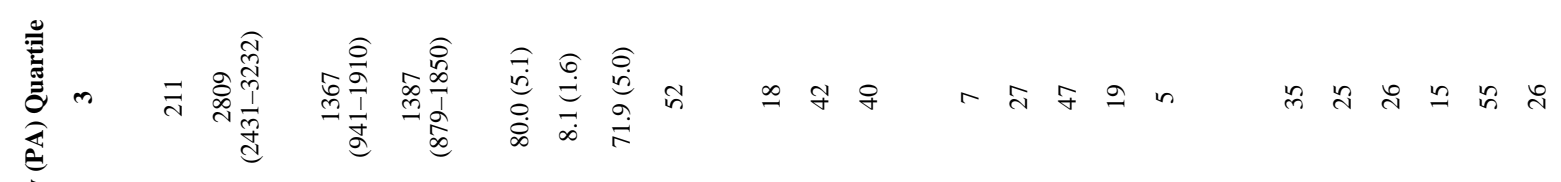

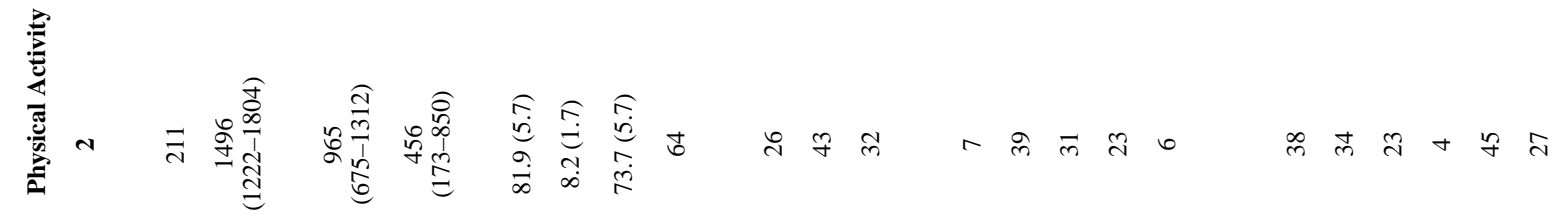

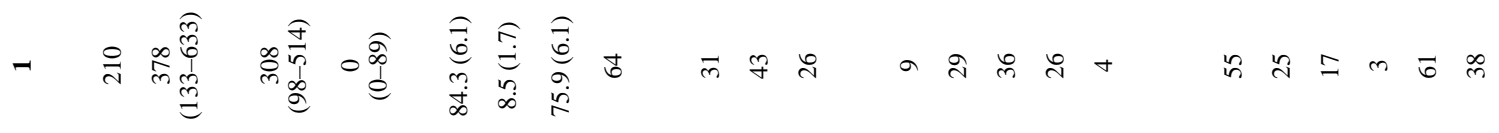


Johnson et al.

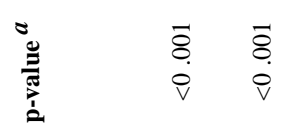

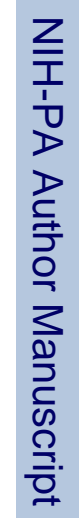

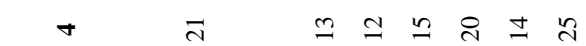

है

$\infty \pm \backsim \pm \pm m$

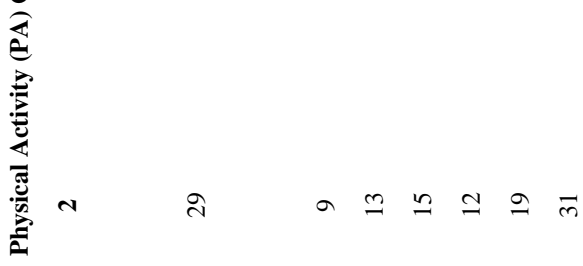

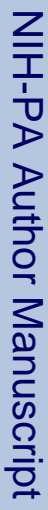

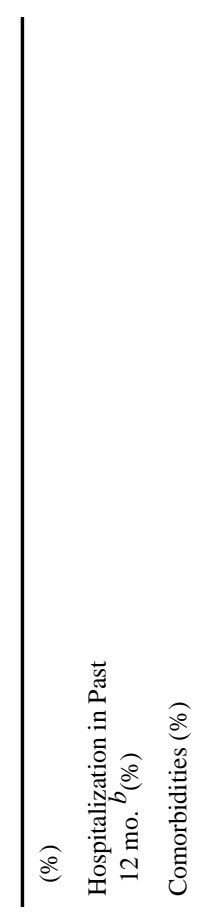

음.

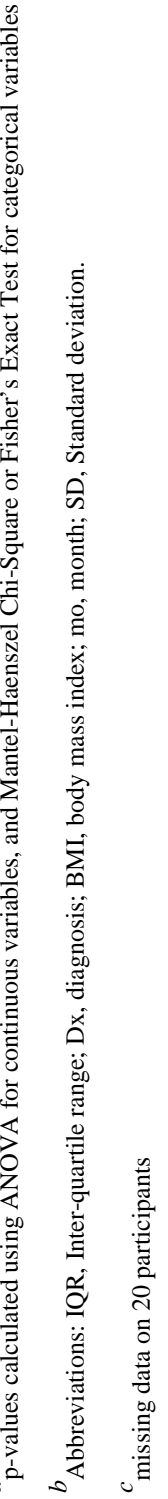


Johnson et al.

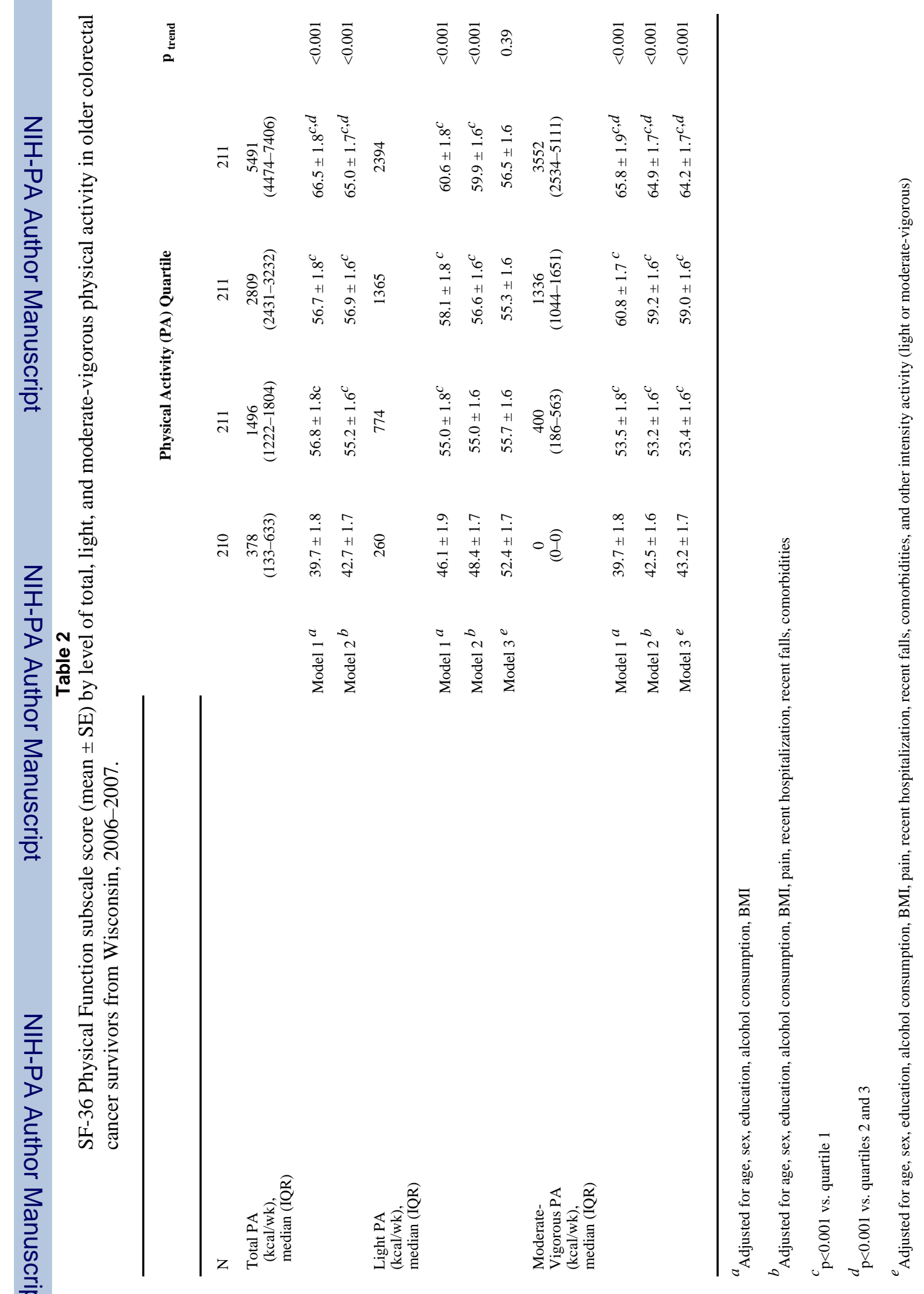




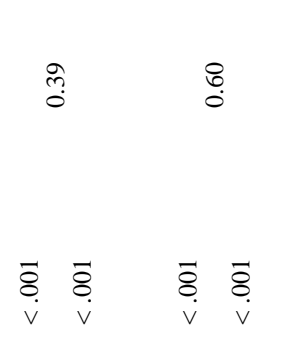

(

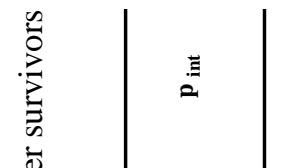




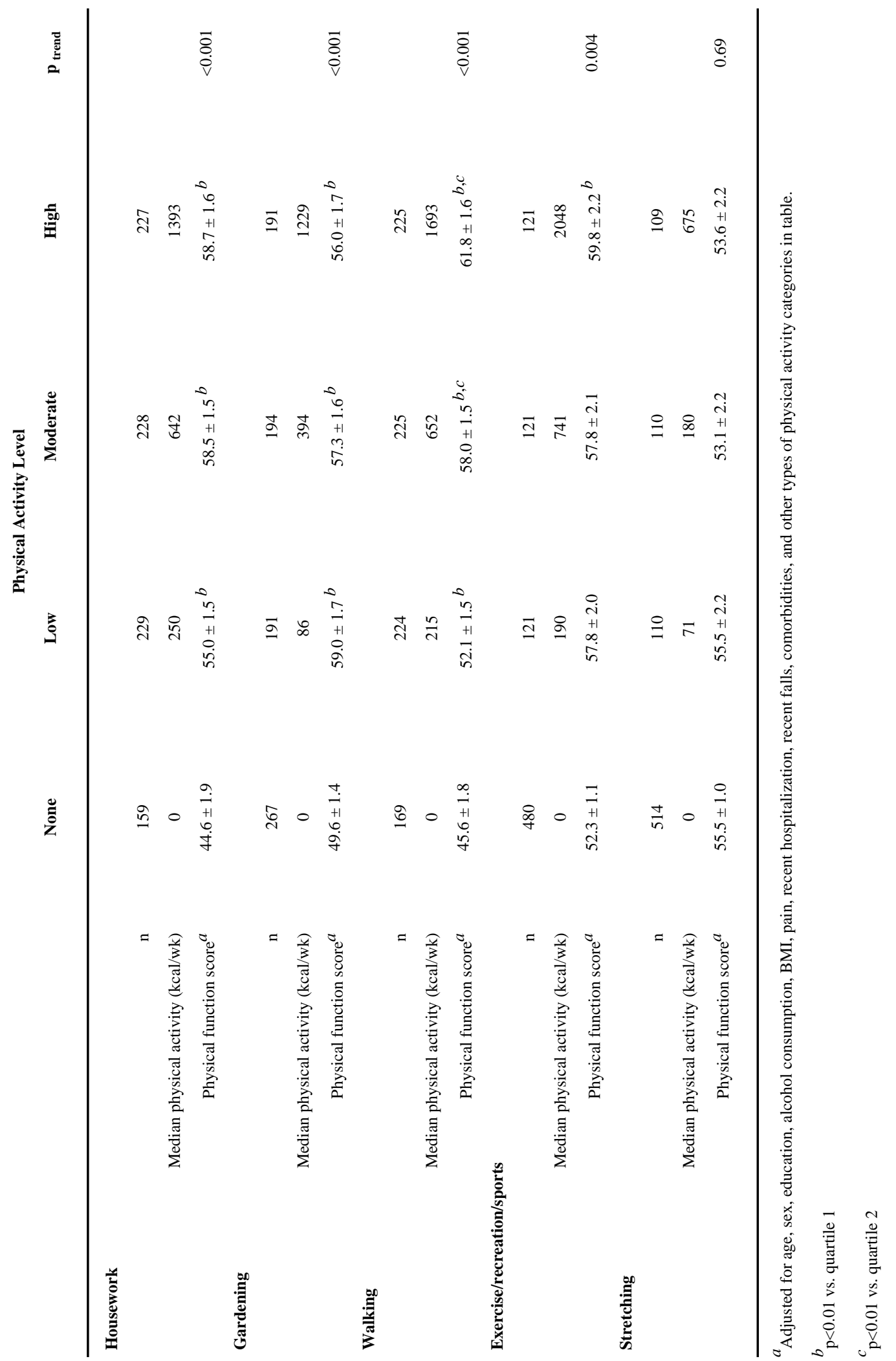

